\title{
Cometary activity of distant object C/2002 VQ94 (LINEAR)
}

\author{
P. P. Korsun ${ }^{1}$, O. V. Ivanova ${ }^{1}$, and V. L. Afanasiev ${ }^{2}$ \\ 1 Main Astronomical Observatory of NAS of Ukraine, Akademika Zabolotnoho 27, 03680 Kyiv, Ukraine \\ e-mail: korsun@mao.kiev.ua \\ 2 Special Astrophysical Observatory of the Russian AS, Nizhnij Arkhyz, 369167, Russia \\ e-mail: vafan@sao.ru
}

Received 4 July 2006 / Accepted 3 August 2006

ABSTRACT

\begin{abstract}
Aims. We have started a program of spectroscopic and photometric investigations of distant active comets in the optical domain. The comets with a significant level of activity-in particular, extended tails-are the objects of our observations.

Methods. The observations were made at the 6-m telescope BTA (SAO RAS, Russia), with the focal reducer SCORPIO attached to the prime focus of the telescope. Long-slit and photometry modes were turned to perform our first observations. The spectral resolution in the spectroscopy mode was $10 \AA$.

Results. The result of the spectrum analysis of comet C/2002 VQ94 (LINEAR) is of particular interest. The comet observed at the heliocentric distance of $6.8 \mathrm{AU}$ clearly shows a rich molecular spectrum. We identified 14 vibrational bands of $\mathrm{CO}^{+}\left(\mathrm{Comet}^{\mathrm{T}} \mathrm{Tal}\right.$ system), emissions of $\mathrm{C}_{3}$, and some tentatively assigned to $\mathrm{N}_{2}^{+}$and $\mathrm{CN}$. It should be emphasized that, for now, $\mathrm{CO}^{+}$and tentatively assigned $\mathrm{N}_{2}^{+}$emissions are detected at a record heliocentric distance.
\end{abstract}

Key words. line: identification - comets: individual: C/2002 VQ94

\section{Introduction}

A new object of 19th magnitude discovered by the LINEAR team on November 11.24 UT, 2002 was reported as an asteroid (Marsden 2002). The heliocentric and geocentric distances were 10.02 AU and 9.16 AU, respectively, at the moment of discovery. With perihelion at $6.8 \mathrm{AU}$, an orbit inclination of $70^{\circ} .5$, and a 3110 year orbit it was assigned as a distant minor planet (Marsden 2003a). The asteroid A/2002 VQ94 (LINEAR) was observed by Tegler et al. (2003) in their continuing photometric survey. Color indices, $B-V=0.92 \pm 0.04, V-R=0.47 \pm 0.02$, $B-R=1.39 \pm 0.05$, were measured on December 31, 2002 . Cometary activity of the object was first detected at the end of August 2003, when the distance from the Sun was 8.9 AU. A prominent $10^{\prime \prime}$ coma with a fanlike morphology spanning p.a. $180-300^{\circ}$ was found on images taken on August 28.5 UT with the University of Hawaii 2.2-m telescope (Green 2003). The object was put in cometary list as C/2002 VQ94 (LINEAR) (Parker 2003). Marsden (2003b) suggests that this is not a "new" comet from the Oort cloud, after analyzing the barycentric values of $1 / \mathrm{a}$

\section{Observations}

We observed comet C/2002 VQ94 (LINEAR) on March 9.88, 2006. The heliocentric distance was 6.80 AU (32 days after perihelion) and the geocentric distance was 5.74 AU (5 days after the closest distance from the Earth). The observations were made at the 6-m telescope BTA (SAO RAS, Russia) with the focal reducer SCORPIO attached to the prime focus of the telescope. Details of the SCORPIO device were described elsewhere by Afanasiev \& Moiseev (2005). We used the SCORPIO in two modes, CCD photometry in broad-band filters and long-slit spectroscopy. A CCD chip
EEV-42-40 of $2048 \times 2048$ pixels was used as the detector. A full field of view of the detector is $6.1^{\prime} \times 6.1^{\prime}$ with an image scale of $0.18^{\prime \prime} /$ pix. In the photometry mode, the comet was observed through $V$ and $R$ filters, while in the spectroscopy mode a long-slit mask with $6.1^{\prime} \times 1.0^{\prime \prime}$ dimensions was inserted into the light cone. Additionally, the transparent grism VPHG550G was used as a disperser. The spectral resolution of our spectra was $10 \AA$ A. Six spectroscopic exposures, each of 300 s duration, were obtained in these observations.

\section{Data reductions}

The raw frames with observed data were reduced in a standard manner. Cleaning from cosmic ray events was the first step in the spectra reductions. Further, we removed bias as the averaged set of the CCD images with zero time exposures. Broad-band images were flat-fielded using observations of the morning sky. Flat-fielding of the spectral observations was performed using spectra of a lamp with smooth variation in energy distribution. Wavelength assignments were made by exposing an He-Ne-Arfilled lamp. To increase the signal/noise ratio of the observed data, we coadded all the images obtained through the $V$ filter and further analyzed the integrated image. The same was done with the images obtained through the $R$ filter and spectroscopic data. Additionally, broad-band images were binned $2 \times 2$ and spectra binned $2 \times 1$, that is, in the slit direction.

Figure 1 is an illustration on the observed data. It is clear that the observed object has a cometary nature. It has a fan-like short tail extended over $60^{\prime \prime}$ and declined from the extended radius vector. It should be pointed out that the morphology of the tail is not typical of distant comets. The tails of distant comets are narrow and slightly curved, without broadening along the tail. 


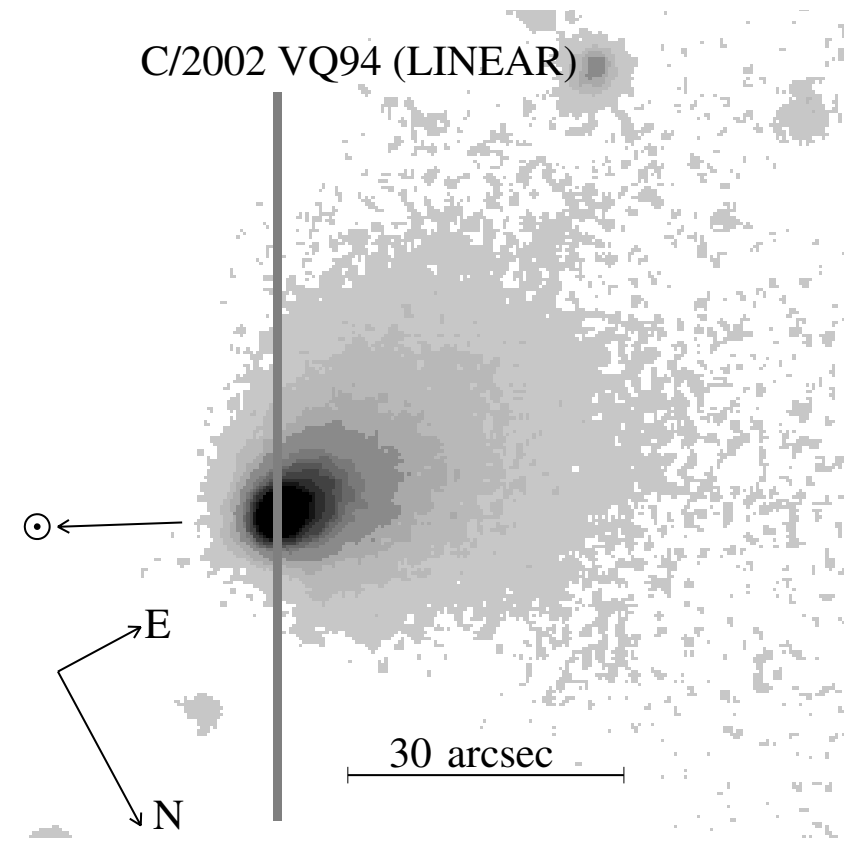

Fig. 1. The $90^{\prime \prime} \times 90^{\prime \prime}$ extraction from the summarized image observed through $V$ and $R$ filters. North, East, and sunward directions are indicated. The vertical line across the comet marks the position of the slit in the spectroscopic mode.

This is not the case. Unfortunately, because of the time limitation on observations, the slit was not oriented along the tail.

To provide photometric calibration of our observations, the star BD+75d325 was observed as a spectrophotometric standard (Oke 1990). The spectral atmospheric transparency at Special Astronomical Observatory has been taken from Kartasheva \& Chunakova (1978). The above-mentioned reductions were performed using programs running under IDL and prepared by Afanasiev. As we have had problems with the photometric calibration of the broad-band observations, we only report here the results of the spectroscopic data analysis.

\section{Results}

Spectroscopic observations of distant objects in the Solar system with cometary activity are not numerous. Only a few emissions of gaseous species have been observed in the optical domain at large distances, beyond 5 AU. Molecular emissions have only been detected in the peculiar periodic comet SchwassmannWachmann $1\left(\mathrm{CO}^{+}, \mathrm{CN}\right)$, giant comet Hale-Bopp $\left(\mathrm{CN}, \mathrm{C}_{3}\right)$, and centaur Chiron $(\mathrm{CN})$.

The aim of our spectroscopic observations was to study the reflectivity of the dust and search for molecular emissions in the coma of distant comets. To obtain the dust reflectivity we divided the observed spectrum by a solar one obtained by convolving our slit function with the Neckel \& Labs (1984) solar spectrum. One can see the result in Fig. 2. The flux ratio comet/Sun shows evidence of the well-defined growth toward red wavelengths. The plot is roughly linear in the 4700-7200 $\AA$ wavelength region with a color gradient of $15 \%$ per $1000 \AA$ assuming normalization at $5500 \AA$.

The surprising thing is that the observed spectrum shows evidently rich molecular emissions. The strongest and the most dispersed along the observed spectral window are the comet-tail bands of $\mathrm{CO}^{+}$. The $(3,0),(2,0),(1,0),(5,1),(3,1),(2,1),(4,2)$, $(3,2),(0,0),(1,1),(2,2),(0,1),(0,2)$, and $(1,2)$ vibrational transitions of the $A^{2} \Pi-X^{2} \Sigma$ band system of $\mathrm{CO}^{+}$are seen clearly in Fig. 2. Their assignments were made using the Ultraviolet and Visible Spectroscopic Database for Atoms and Molecules in Celestial Objects compiled by $\operatorname{Kim}(1994,1998)$ and fluorescence calculations by Arpigny (1964) and Magnani \& A'Hearn (1986).

The $(3,0)$ band of $\mathrm{CO}^{+}$is contaminated by the $(020-000)$ vibrational band of the $\mathrm{C}_{3}$ emissions. The (000-000) and (000020) vibrational bands of the ${ }^{1} \Pi_{u}-\Sigma_{g}^{+}$transition are present as well (Gausset et al. 1965).

The broad spectral feature with local maxima at $3884 \AA$ and $3904 \AA$ coincides with the $(5,1)$ vibrational band of $\mathrm{CO}^{+}$. Nevertheless, the intensity of the $(5,1)$ band is much lower so as to fit the feature. Two additional emissions, namely, the $(0,0)$ vibrational band of the $\mathrm{CN}$ violet system and the $(0,0)$ band of $\mathrm{N}_{2}^{+}$, can resolve the problem. The presence of the $\mathrm{CN}$ emissions looks natural, as it is observed in comets at large heliocentric distances, while the presence of $\mathrm{N}_{2}^{+}$is questionable and will be discussed in the next section. We consider the above-mentioned identification of $\mathrm{CN}$ and $\mathrm{N}_{2}^{+}$as tentative assignments. To assign them we used the LIFBASE software (Luque \& Crosley 1999). Other emissions, which are undoubtedly present in the spectrum, remain unassigned.

\section{Discussion}

We observed a distant comet with an unusual spectroscopic appearance. The obtained spectrum is rich in $\mathrm{CO}^{+}$. Fourteen emission bands of the comet-tail bands of $\mathrm{CO}^{+}$are detected at the heliocentric distance of $6.8 \mathrm{AU}$ in the observed spectral window. So far, $\mathrm{CO}^{+}$beyond Jupiter's orbit has been recorded in the peculiar periodic comet 29P/Schwassmann-Wachmann 1, at $r \leq 6.2 \mathrm{AU}$ (Larson 1980; Cochran et al. 1982). Moreover, only the two strongest bands of $\mathrm{CO}^{+},(2,0)$ and $(3,0)$, have been assigned in the later spectra.

There was only once when the $\mathrm{C}_{3}$ emissions were observed at large heliocentric distances, namely, at 7.0 AU in Comet HaleBopp (Rauer et al. 2003). There is no doubt that we detect the $\mathrm{C}_{3}$ emissions, too, as three features in our spectra are coincident with the strongest (000-000), (000-020), and (020-000) vibrational transitions of the ${ }^{1} \Pi_{u}-\Sigma_{g}^{+}$system of $\mathrm{C}_{3}$.

We consider the $\mathrm{CN}$ and $\mathrm{N}_{2}^{+}$emissions to be tentatively assigned. There is no surprise for the $\mathrm{CN}$ assignment, as it was previously observed in comets at larger heliocentric distances. $\mathrm{CN}$ has been observed in Chiron at a record heliocentric distance, 11.3 AU (Bus et al. 1991), and at 9.8 AU in Comet Hale-Bopp (Rauer et al. 2003).

Some uncertainty exists in the $\mathrm{N}_{2}^{+}$case. The $\mathrm{N}_{2}^{+}$emissions are rarely observed in comets. Lutz et al. (1993) and Wyckoff \& Theobald (1989) report detecting $\mathrm{N}_{2}^{+}$in the tails of comets Halley and C/1987 P1 (Bradfild). While Cochran et al. (1992, 2000, 2002) has evidence of three comets for which no $\mathrm{N}_{2}^{+}$was detected in high-resolution spectra. Arpigny examined collections of photographic spectra (Swigs \& Haser 1956) and other data at his disposal and estimated the intensity ratio for $\mathrm{N}_{2}^{+} / \mathrm{CO}^{+}$in 12 comets (Cochran et al. 2000). The record detection of $\mathrm{N}_{2}^{+}$was reported for comet Humason (1962 VIII) at 2.6 AU (Greenstein \& Arpigny 1962).

Our spectrum was checked carefully against a potential confusion with $\mathrm{N}_{2}^{+}$emissions from airglow. We observed a long-slit spectrum. The height of the slit was $6.1^{\prime}$, whereas the coma of the comet was extended along the slit less than $1^{\prime}$. Thus, we examined the spectrum where it was free of the cometary emissions 


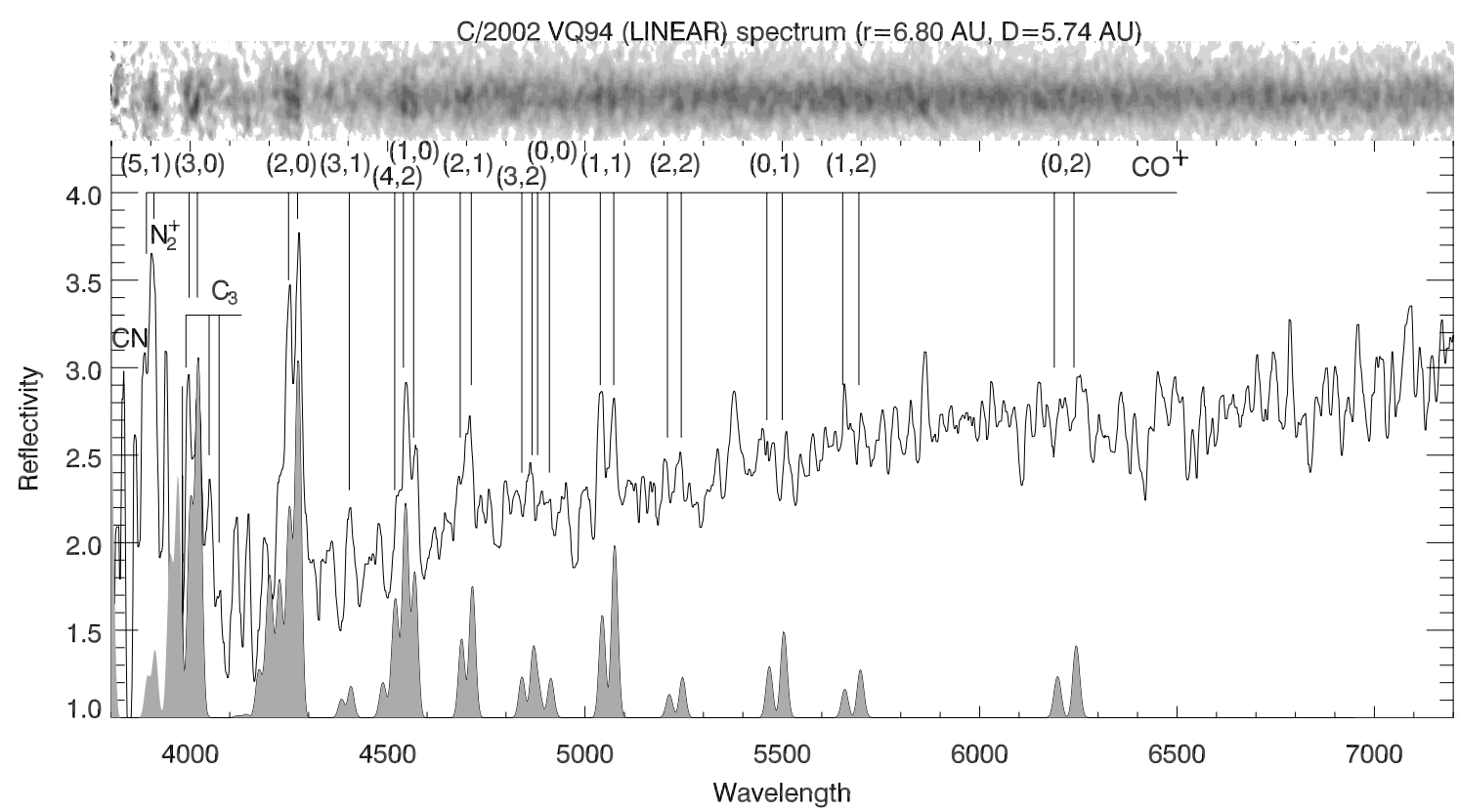

Fig. 2. Observed spectrum and flux ratio plot comet/Sun for C/2002 VQ94 (LINEAR). Assigned molecular emissions are marked. Filled features at the bottom of the plot show the $\mathrm{CO}^{+}$spectrum calculated under the Boltzmann approximation (Kim 1994, 1998).

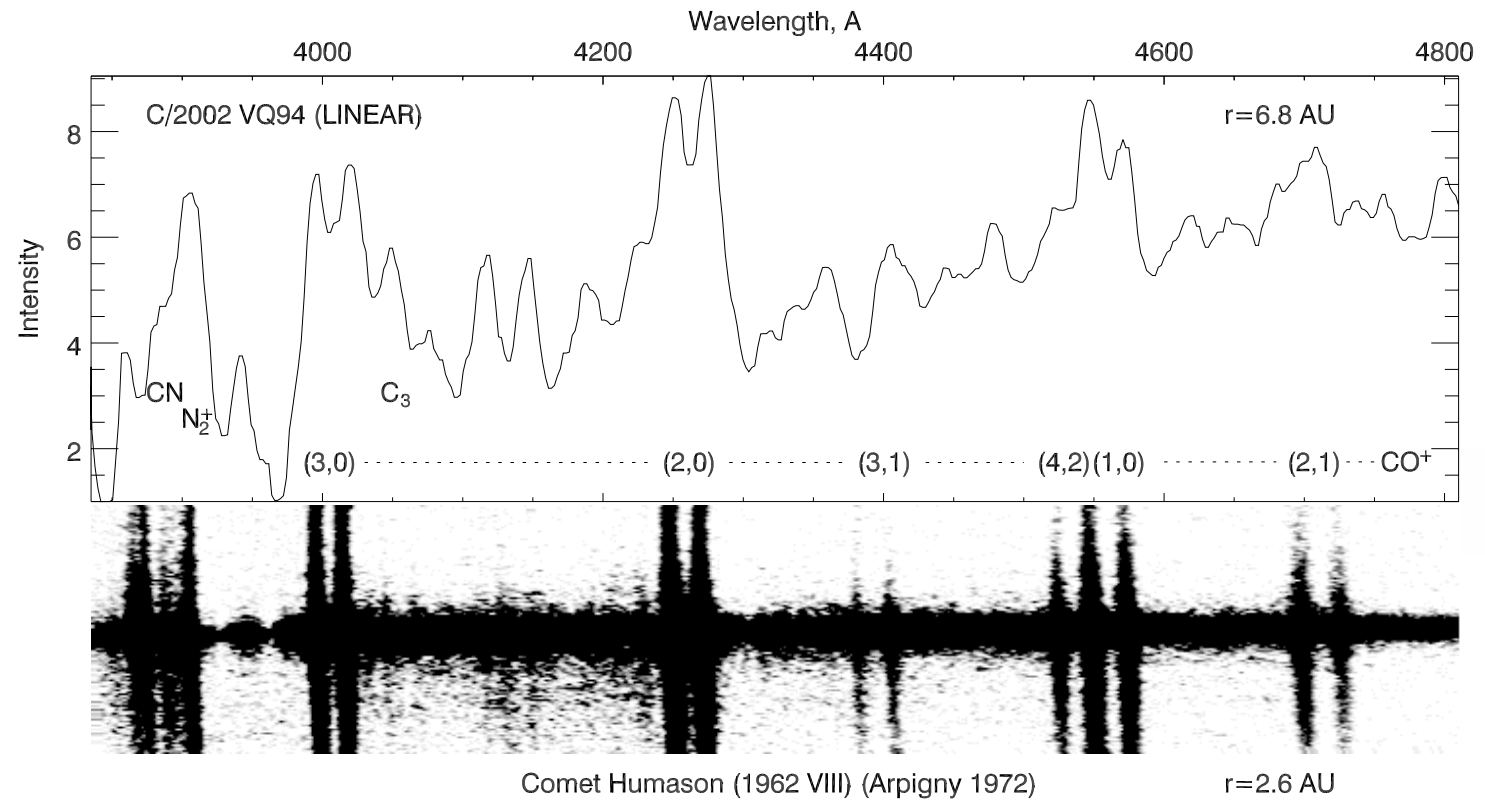

Fig. 3. Spectrum of comet C/2002 VQ94 (LINEAR) (top) is displayed together with a part of the comet Humason (1962 VIII) spectrogram (bottom) to compare available molecular emissions. The spectrogram of comet Humason is taken from Arpigny (1972). Identifications of principal features are indicated as well.

and found no detectable spectral feature near $3910 \AA$ A. Cochran (2002) reviewed the problem of terrestrial $\mathrm{N}_{2}^{+}$and argues that telluric $\mathrm{N}_{2}^{+}$can only be found in dayglow spectra, while we made the spectroscopic observations when it was astronomical night.

Additional arguments in favor of the identification of $\mathrm{N}_{2}^{+}$in the spectrum of comet C/2002 VQ94 (LINEAR) are the following speculations. Hill et al. (2001) argue that comets, formed early in nebular history, should be rich in $\mathrm{CO}, \mathrm{CO}_{2}, \mathrm{~N}_{2}$, and amorphous dust. We consider that the discussed here comets are CO-rich objects formed under similar conditions in the Solar nebula: Morehouse (1908 II), Humason (1962 VIII), and C/2002 VQ94 (LINEAR). They show essential distinctions when comparing their spectra with typical cometary spectra where the neutral molecules are much more abundant than $\mathrm{CO}^{+}$(Swigs \& Haser 1956). The spectra of CO-rich comets show the significant predominance of $\mathrm{CO}^{+}$, the strong emissions of $\mathrm{CN}$ and $\mathrm{N}_{2}^{+}$, and the measurable $\mathrm{C}_{3}$ features. It should also be emphasized that the comets mentioned above show similar spectroscopic features at significantly different heliocentric distances (1.4 AU for Comet Morehouse (1908 II) (Fowler 1912), 2.6 AU for comet Humason (1962 VIII) (Greenstein \& Arpigny 1962), and 6.8 AU for C/2002 VQ94 (LINEAR)). Figure 3 is a convincing illustration of our discussion, reproducing relative intensity plot of the observed spectrum of comet C/2002 VQ94 (LINEAR) and part of the Palomar plate with the spectrum of comet Humason (1962 VIII) (Arpigny 1972), where they are overlapping. 
Estimating $\mathrm{N}_{2}^{+} / \mathrm{CO}^{+}$is of fundamental importance. To derive the ratio we should measure intensities of the $\mathrm{CO}^{+}$and $\mathrm{N}_{2}^{+}$emissions. First, a scaled solar spectrum was subtracted from the observed one. Further, the rest continuum caused by the wavelength efficiency of the solar-light scattering by the cometary dust was removed by subtracting a low-frequency polynomial from the previous result. The detected $\mathrm{N}_{2}^{+}$is contaminated by the $\mathrm{CO}^{+}(5,1)$ and $\mathrm{CN}(0,0)$ bands. To estimate the contribution of $\mathrm{N}_{2}^{+}$to the low-resolution feature, we modeled the $\mathrm{CO}^{+}(5,1)$ and $\mathrm{CN}(0,0)$ emissions using laboratory data. As for the $\mathrm{CO}^{+}$emissions, we measured the $(2,0)$ band.

Once the band intensity is known, the column density can be computed using

$N=L / g_{v^{\prime} v^{\prime \prime}}$,

where $N$ is the column density, $L$ the integrated band intensity, and $g_{v^{\prime} v^{\prime \prime}}$ the excitation factor. We used excitations factors of $7.0 \times 10^{-2}$ photons $\mathrm{s}^{-1} \mathrm{~mol}^{-1}$ for the $\mathrm{N}_{2}^{+}(0,0)$ band (Lutz et al. $1993)$ and $3.55 \times 10^{-3}$ photons $\mathrm{s}^{-1} \mathrm{~mol}^{-1}$ for the $\mathrm{CO}^{+}(2,0)$ band (Lutz et al. 1993). Using

$\frac{\mathrm{N}_{2}^{+}}{\mathrm{CO}^{+}}=\frac{g_{\mathrm{CO}^{+}}}{g_{\mathrm{N}_{2}^{+}}} \frac{L_{\mathrm{N}_{2}^{+}}}{L_{\mathrm{CO}^{+}}}$

we derived a value of $\mathrm{N}_{2}^{+} / \mathrm{CO}^{+}=0.04$. The value is the same order as for comets Morehouse (1908 II), Humason (1962 VIII), and it agrees with Arpigny's estimation of the intensity ratio for $\mathrm{N}_{2}^{+} / \mathrm{CO}^{+}$(Cochran et al. 2000). Additionally, the ratio correlates with the experimental results of measuring the amounts of $\mathrm{N}_{2}$ and $\mathrm{CO}$, which are trapped in the ice at low temperatures (Notesco \& Bur-Nun 1996). However, the presence of amorphous water ice is not obvious due to the possible mixing of higher temperature material and low-temperature ices in the early solar nebula (Kemper et al. 2004; Lisse et al. 2006).

\section{Conclusions}

1. An emission-rich comet was observed at the heliocentric distance of $6.8 \mathrm{AU} . \mathrm{CO}^{+}, \mathrm{C}_{3}$ emissions detected, and $\mathrm{N}_{2}^{+}$and $\mathrm{CN}$ emissions tentatively assigned in the spectrum of comet C/2002 VQ94 (LINEAR).

2. We suggest a group of the CO-rich comets with similar molecular emission spectra that were formed under similar conditions in the Solar nebula. The significant predominance of $\mathrm{CO}^{+}$and the strong emissions of $\mathrm{CN}, \mathrm{N}_{2}^{+}$, and $\mathrm{C}_{3}$ are their distinguishing spectroscopic characteristics.
3. The value of $\mathrm{N}_{2}^{+} / \mathrm{CO}^{+}=0.04$ agrees well with experimental results of trapping $\mathrm{CO}$ and $\mathrm{N}_{2}$ in amorphous ice, which supports the low-temperature formation of this comet. However, there are other mechanisms that can produce the observed $\mathrm{CO}$ and $\mathrm{N}_{2}$ abundances.

Acknowledgements. The observations were performed thanks to the support of the Schedule Committee for Large Telescopes (Russian Federation).

\section{References}

Afanasiev, V. L., \& Moiseev, A. V. 2005, Astr. Lett., 31, 193

Arpigny, C. 1964, Thesis for the Degree of Doctor of Philosophy, California Institute of Technology, Pasadena, California

Arpigny, C. 1972, in Proc. of Tucson Comet Conf., Comets: Scientific Data and Missions, ed. G. P. Kuiper, \& E. Roemer, Tucson, Arizona

Bus, S. J., A'Hearn, M. F., Schleicher, D. G., \& Bowell, E. 1991, Science, 251, 774

Cochran, A. L. 2002, ApJ, 576, L165

Cochran, A. L., Cochran, W. D., \& Barker, E. S. 1982, ApJ, 254, 816

Cochran, A. L., Cochran, W. D., Ramseyer, T. F., \& Storrs, A. D. 1992, Icarus, 98, 151

Cochran, A. L., Cochran, W. D., \& Barker, E. S. 2000, Icarus, 146, 583

Gausset, L., Herzberg, G., Lagerqvist, A., \& Rosen, B. 1965, ApJ, 142, 45

Green, D. W. E. 2003, IAUC 8194

Greenstein, J. L., \& Arpigny, C. 1962, ApJ, 135, 892

Fowler, A. 1912, ApJ, 35, 85

Hill, H. G. M., Grady, C. A., Ill, J. A. N., et al. 2001, PNASS, 98, 2182

Kartasheva, T. A., \& Chunakova, N. M. 1978, Izvestija SAO, 10, 44 (Rus.)

Kemper, F., Vriend, W. J., \& Tielens, A. G. G. M. 2004, ApJ, 609, 826

Kim, S. J. 1994, J. Korean Astron. Soc., 111, 11

Kim, S. J. 1998, private communication

Larson, S. M. 1980, ApJ, 238L, 47L

Lisse, C. M., VanCleve, J., Adams, A. C., et al. 2006, Science, in press

Luque, J., \& Crosley, D. R. 1999, SRI International Report MP 99-009

Lutz, B. L., Womack, M., \& Wagner, R. M. 1993, ApJ, 407, 402

Magnani, L., \& A'Hearn 1986, ApJ, 302, 477

Marsden, B. G. 2002, MPEC 2002-V71

Marsden, B. G. 2003a, MPEC 2003-D05

Marsden, B. G. 2003b, MPEC 2003-R43

Neckel, H., \& Labs, D. 1984, Sol. Phys., 90, 205

Notesco, G., \& Bar-Nun, A. 1996, Icarus, 122, 118

Oke, J. B. 1990, AJ, 99, 1621

Parker, J. W. 2003, Distant ECOs. The Kuiper Belt Electronic Newsletter, No. 32

Rauer, H., Helbert, J., Arpigny, C., et al. 2003, A\&A, 397, 1109

Rehfuss, B. D., Suh, M.-H., Miller, T. A., \& Bodybey, V. E. 1992, J. Mol. Sp., 151,437

Swings, P., \& Haser, L. 1956, Atlas of Representative Cometary Spectra, Louvain, Belgium

Tegler, S. C., Romanishin, W., \& Consolmagno, G. J. 2003, ApJ, 599, L49

Wyckoff, S., \& Theobald, J. 1989, Adv. Space Res., 9, 157 